Subscriber access provided by Caltech Library

\title{
Article
}

\section{Linear-response time-dependent embedded mean-field theory}

\author{
Feizhi Ding, Takashi Tsuchiya, Frederick R. Manby, and Thomas Francis Miller
}

J. Chem. Theory Comput., Just Accepted Manuscript • DOI: 10.1021/acs.jctc.7b00666 • Publication Date (Web): 07 Aug 2017

Downloaded from http://pubs.acs.org on August 15, 2017

\section{Just Accepted}

"Just Accepted" manuscripts have been peer-reviewed and accepted for publication. They are posted online prior to technical editing, formatting for publication and author proofing. The American Chemical Society provides "Just Accepted" as a free service to the research community to expedite the dissemination of scientific material as soon as possible after acceptance. "Just Accepted" manuscripts appear in full in PDF format accompanied by an HTML abstract. "Just Accepted" manuscripts have been fully peer reviewed, but should not be considered the official version of record. They are accessible to all readers and citable by the Digital Object Identifier (DOI®). "Just Accepted" is an optional service offered to authors. Therefore, the "Just Accepted" Web site may not include all articles that will be published in the journal. After a manuscript is technically edited and formatted, it will be removed from the "Just Accepted" Web site and published as an ASAP article. Note that technical editing may introduce minor changes to the manuscript text and/or graphics which could affect content, and all legal disclaimers and ethical guidelines that apply to the journal pertain. ACS cannot be held responsible for errors or consequences arising from the use of information contained in these "Just Accepted" manuscripts. 


\title{
Linear-response time-dependent embedded
}

\section{mean-field theory}

\author{
Feizhi Ding, ${ }^{\dagger}$ Takashi Tsuchiya, ${ }^{\ddagger}$ Frederick R. Manby, ${ }^{\ddagger}$ and Thomas F. Miller III*,† \\ $\dagger$ Division of Chemistry and Chemical Engineering, California Institute of Technology, \\ Pasadena, CA 91125, USA \\ $\ddagger$ Centre for Computational Chemistry, School of Chemistry, University of Bristol, Bristol \\ BS8 1TS, UK \\ E-mail: tfm@caltech.edu
}

\begin{abstract}
We present a time-dependent (TD) linear-response description of excited electronic states within the framework of embedded mean-field theory (EMFT). TD-EMFT allows for subsystems to be described at different mean-field levels of theory, enabling straightforward treatment of excited-states and transition properties. We provide benchmark demonstrations of TD-EMFT for both local and non-local excitations in organic molecules, as well as applications to chlorophyll a, solvatochromic shifts of a dye in solution, and sulfur K-edge X-ray absorption spectroscopy (XAS). It is found that mixed-basis implementations of TD-EMFT lead to substantial errors in terms of transition properties; however, as previously found for ground-state EMFT, these errors are largely eliminated with the use of Fock-matrix corrections. These results indicate that TD-EMFT is a promising method for the efficient, multi-level description of excitedstate electronic structure and dynamics in complex systems.
\end{abstract}




\section{Introduction}

Excited electronic states are central to photo-induced processes in a wide variety of chemical, biological, and materials applications. In any molecular system, the choice of electronic structure methods for calculating excited-state properties involves a necessary compromise between accuracy and computational cost. Despite its shortcomings, ${ }^{1-4}$ time-dependent density functional theory (TDDFT) offers an appealing balance between these factors and is thus widely used; and yet the costly scaling of TDDFT (formally $\mathcal{O}\left(N^{4}\right)$ ) makes the approach unaffordable for many large-scale applications.

Various strategies have been developed to expand the applicability of excited-state methods. For example, linear-scaling implementations based on linear response take advantage of spatial locality of either the atomic ${ }^{5-7}$ or molecular ${ }^{8-13}$ orbitals. An alternative strategy employs subsystem embedding to describe localized excitations, including TDDFT implementations using either fragment molecular orbitals ${ }^{14}$ or frozen-density embedding, ${ }^{15-20}$ as well as the QM/MM approach. ${ }^{21-24}$

While each of these methods has merits, they also have limitations. For example, methods based on localized molecular orbitals lead to complicated implementations for analytical gradients and properties, while many embedding methods place constraints on the subsystem particle numbers, spin state, and spatial extent of the excitation, or they neglect particle-number fluctuations between subsystems, or the environmental response to the excitation. Removing such constraints has motivated the recent development of embedding strategies that are formally exact in the description of subsystem interactions ${ }^{25-37}$ and allow for particle-number fluctuations between subsystems via their description as open quantum systems. ${ }^{35-37}$

Here, we introduce time-dependent embedded mean-field theory (TD-EMFT), a linear-response approach to describe excited electronic states using the EMFT framework. ${ }^{37,38}$ TD-EMFT provides subsystem embedding at different levels of mean-field theory, avoiding the need to specify or fix the particle number or spin state for each subsystem. It is simple to implement, and because EMFT is itself a mean-field theory, 
calculation of analytical nuclear gradients and response properties remains straightforward. We demonstrate TD-EMFT for a range of benchmark systems and applications, both with and without Fock-matrix corrections, ${ }^{39}$ illustrating that TD-EMFT allows for comparable accuracy to TDDFT with the potential for vastly reduced computational cost. This work is complementary to the recent extension of EMFT in terms of the real-time TD-DFT formalism by Parkhill and coworkers. ${ }^{40}$

\section{Methodology}

In this section, we first review EMFT and present the linear-response formulation. Throughout the discussion, we use restricted closed-shell orbitals for simplicity; generalization to spin-unrestricted cases is straightforward. As usual, indices $i, j, k, l$ denote occupied orbitals; $a, b, c, d$ unoccupied orbitals, and $p, q, r, s$ arbitrary molecular orbitals.

\subsection{Embedded Mean-Field Theory}

EMFT employs a partitioning of the one-particle reduced density matrix in the representation of an atomic-orbital (AO) basis set ${ }^{37}$ (or a block-orthogonalized AO basis ${ }^{38}$ ):

$$
\mathbf{D}=\left(\begin{array}{ll}
\mathbf{D}^{\mathrm{AA}} & \mathbf{D}^{\mathrm{AB}} \\
\mathbf{D}^{\mathrm{BA}} & \mathbf{D}^{\mathrm{BB}}
\end{array}\right),
$$

where $\mathbf{D}^{\mathrm{AA}}$ and $\mathbf{D}^{\mathrm{BB}}$ denote the density-matrix blocks that belong to subsystems $\mathrm{A}$ and B, respectively. The EMFT energy functional associated with describing subsystems A and $\mathrm{B}$ with these two different levels of mean-field theory is given by

$$
E^{\mathrm{EMFT}}[\mathbf{D}]=E^{(2)}[\mathbf{D}]+E^{(1)}\left[\mathbf{D}^{\mathrm{AA}}\right]-E^{(2)}\left[\mathbf{D}^{\mathrm{AA}}\right]
$$

where $E^{(1)}$ and $E^{(2)}$ are the different mean-field theory energy functionals. The EMFT ground state energy is then obtained by minimizing $E^{\text {EMFT }}$ with respect to $\mathbf{D}$, while enforcing the idempotency and normalization constraints for the total density matrix. 
This leads to the familiar self-consistent field (SCF) equation

$$
\mathbf{F C}=\mathrm{SC} \varepsilon
$$

where the EMFT Fock matrix $\mathbf{F}$ is the derivative of $E^{\mathrm{EMFT}}$ with respect to the total density matrix; $\mathbf{C}$ is the molecular orbital (MO) coefficient matrix, and $\varepsilon$ is the diagonal matrix containing the MO energies in the canonical representation.

EMFT provides a general framework for quantum embedding at the mean-field level. Examples include the embedding of relatively high-cost mean-field methods such as DFT with hybrid, double-hybrid, or range-separated exchange-correlation (xc) functionals into low-cost methods such as minimal-basis DFT with local-density approximation (LDA), and density functional tight-binding (DFTB) models.

\subsubsection{Fock-corrected density functional theory}

For ground-state EMFT applications, the use of Fock-matrix corrections ${ }^{39}$ has been shown to eliminate errors associated with mismatches between the high- and low-level theories. ${ }^{39,41}$ The same strategy will be employed here in the context of electronic excited states. Fock-corrected density functional theory (FCDFT) ${ }^{39}$ employs parameterized Fock-matrix corrections to KS-DFT with a low-cost xc functional (such as LDA) and a minimal basis (such as STO- $3 \mathrm{G}^{42}$ ), to approximate KS-DFT results obtained with more sophisticated functional (such as B3LYP) and larger basis. ${ }^{39}$

The FCDFT energy functional is

$$
E^{\mathrm{FCDFT}}[\mathbf{D}]=E^{\mathrm{DFT}}[\mathbf{D}]+\operatorname{Tr}(\mathbf{D L})+U_{\text {cor }},
$$

where $E^{\mathrm{DFT}}[\mathbf{D}]$ is the DFT functional of the minimal-basis one-particle density matrix D evaluated, for example, at the LDA/STO-3G level. The term $U_{\text {cor }}$ is a sum of shortranged pairwise interactions that gives rise to the correction to the potential energy. 
Lastly, $\mathbf{L}$ is the Fock correction matrix, which is defined as

$$
L_{\mu \nu}= \begin{cases}\delta_{\mu \nu} \epsilon_{\mu}, & \mu, \nu \in \text { atom } I \\ F_{\mu \nu}^{\mathrm{SK}}\left(\mathbf{R}_{I J}\right), & \mu \in \text { atom } I, \nu \in \text { atom } J, I \neq J\end{cases}
$$

where $\epsilon_{\mu}$ are fitted, atom-specific diagonal energy shifts; $F_{\mu \nu}^{\mathrm{SK}}\left(\mathbf{R}_{I J}\right)$ are fitted atomatom interaction functions. Minimization of the energy functional in Eq. 4 with respect to $\mathbf{D}$ ensures that FCDFT provides a self-consistent correction to the low-cost meanfield theory with energy $E^{\mathrm{DFT}}[\mathbf{D}]$. Full details of the FCDFT method is given in Ref. 39, and additional analysis of its performance for ground-state problems is given in Ref. 41.

In the current work, we employ the FCLDA implementation of FCDFT in which $E^{\mathrm{DFT}}[\mathbf{D}]$ in Eq. (4) corresponds to the LDA/STO-3G level. The FCDFT parameters and fitting procedure are described in the Computational Details section. The term $U_{\text {cor }}$ is neglected since it does not affect the MOs or vertical excitation energies.

For EMFT calculations using FCDFT in subsystem B, the ground state density is obtained by minimizing the following energy expression ${ }^{39}$

$$
E^{\mathrm{DFT}-\mathrm{in}-\mathrm{FCDFT}}[\mathbf{D}]=E^{(2)}[\mathbf{D}]+E^{(1)}\left[\mathbf{D}^{\mathrm{AA}}\right]-E^{(2)}\left[\mathbf{D}^{\mathrm{AA}}\right]+\operatorname{Tr}(\mathbf{D L})-\operatorname{Tr}\left(\mathbf{D}^{\mathrm{AA}} \mathbf{L}^{\mathrm{AA}}\right)
$$

where the Fock correction matrix $\mathbf{L}$ is projected from the minimal-basis for the low-level subsystem to the basis for the whole system, as described in Ref. 39.

\subsection{Time-dependent EMFT}

Here, we briefly present the derivation of TD-EMFT, which closely follows that of conventional linear-response TDDFT. ${ }^{43}$ The presented equations assume that the meanfield reference state is computed by EMFT, using AO partitioning and assuming that the mean-field theories correspond to pure DFT functionals. Generalization to other cases (such as using a hybrid DFT functional or FCDFT) and other subsystem-partitioning 
schemes ${ }^{38}$ can be straightforwardly obtained, by applying linear response ${ }^{43}$ to the corresponding EMFT equations.

We consider the linear response of the electron density for the EMFT reference state, $\delta \rho$, to an effective one-particle perturbation, $\delta v$ of frequency $\omega$,

$$
\delta \rho(\mathbf{r}, \omega)=\int d \mathbf{r}^{\prime} \chi^{\mathrm{s}}\left(\mathbf{r}, \mathbf{r}^{\prime}, \omega\right) \delta v\left(\mathbf{r}^{\prime}, \omega\right)
$$

Here, $\delta v$ is defined as

$$
\begin{aligned}
\delta v(\mathbf{r}, \omega)= & \delta v_{\mathrm{ext}}(\mathbf{r}, \omega)+\delta v_{\mathrm{H}}(\mathbf{r}, \omega)+\int d \mathbf{r}^{\prime} f_{\mathrm{xc}}^{(2)}\left(\mathbf{r}, \mathbf{r}^{\prime}, \omega\right) \delta \rho\left(\mathbf{r}^{\prime}, \omega\right) \\
& +\int d \mathbf{r}^{\prime} f_{\mathrm{xc}, \mathrm{AA}}^{(1)}\left(\mathbf{r}, \mathbf{r}^{\prime}, \omega\right) \delta \rho_{\mathrm{A}}\left(\mathbf{r}^{\prime}, \omega\right)-\int d \mathbf{r}^{\prime} f_{\mathrm{xc}, \mathrm{AA}}^{(2)}\left(\mathbf{r}, \mathbf{r}^{\prime}, \omega\right) \delta \rho_{\mathrm{A}}\left(\mathbf{r}^{\prime}, \omega\right),
\end{aligned}
$$

in which $\delta v_{\text {ext }}$ represents the external perturbation, and $\delta v_{\mathrm{H}}$ is the first-order change in the Coulomb potential. The last three terms in Eq. 8 correspond to the first-order changes in the xc potential for the low-level functional on the entire system, the highlevel functional on subsystem A, and the low-level functional on subsystem A, respectively; although the xc kernel in each of these terms (i.e., $f_{\mathrm{xc}}^{(2)}\left(\mathbf{r}, \mathbf{r}^{\prime}, \omega\right), f_{\mathrm{xc}, \mathrm{AA}}^{(1)}\left(\mathbf{r}, \mathbf{r}^{\prime}, \omega\right)$, and $\left.f_{\mathrm{xc}, \mathrm{AA}}^{(2)}\left(\mathbf{r}, \mathbf{r}^{\prime}, \omega\right)\right)$ is generally frequency dependent, we will neglect this dependence by employing the adiabatic approximation. ${ }^{4}$ As a result, the low-level xc kernel, $f_{\mathrm{xc}}^{(2)}\left(\mathbf{r}, \mathbf{r}^{\prime}\right)$, is obtained from the second derivative of the low-level xc functional with respect to the total density,

$$
f_{\mathrm{xc}}^{(2)}\left(\mathbf{r}, \mathbf{r}^{\prime}\right)=\frac{\delta^{2} E_{\mathrm{xc}}^{(2)}[\rho]}{\delta \rho(\mathbf{r}) \delta \rho\left(\mathbf{r}^{\prime}\right)}
$$

and $f_{\mathrm{xc}, \mathrm{AA}}^{(1)}\left(\mathbf{r}, \mathbf{r}^{\prime}\right)$ and $f_{\mathrm{xc}, \mathrm{AA}}^{(2)}\left(\mathbf{r}, \mathbf{r}^{\prime}\right)$ are the second derivatives of the high- and low-level functionals with respect to the subsystem A density, respectively:

$$
f_{\mathrm{xc}, \mathrm{AA}}^{(1)}\left(\mathbf{r}, \mathbf{r}^{\prime}\right)=\frac{\delta^{2} E_{\mathrm{xc}}^{(1)}\left[\rho_{\mathrm{A}}\right]}{\delta \rho_{\mathrm{A}}(\mathbf{r}) \delta \rho_{\mathrm{A}}\left(\mathbf{r}^{\prime}\right)}, \quad f_{\mathrm{xc}, \mathrm{AA}}^{(2)}\left(\mathbf{r}, \mathbf{r}^{\prime}\right)=\frac{\delta^{2} E_{\mathrm{xc}}^{(2)}\left[\rho_{\mathrm{A}}\right]}{\delta \rho_{\mathrm{A}}(\mathbf{r}) \delta \rho_{\mathrm{A}}\left(\mathbf{r}^{\prime}\right)}
$$


The changes in the total density and subsystem A density are

$$
\begin{aligned}
\delta \rho(\mathbf{r}, \omega) & =\sum_{i, a}\left[\delta D_{i a}(\omega) \psi_{i}(\mathbf{r}) \psi_{a}^{*}(\mathbf{r})+\delta D_{a i}(\omega) \psi_{a}(\mathbf{r}) \psi_{i}^{*}(\mathbf{r})\right] \\
\delta \rho_{\mathrm{A}}(\mathbf{r}, \omega) & =\sum_{i, a}\left[\delta D_{i a}(\omega) \psi_{i}^{\mathrm{A}}(\mathbf{r}) \psi_{a}^{\mathrm{A} *}(\mathbf{r})+\delta D_{a i}(\omega) \psi_{a}^{\mathrm{A}}(\mathbf{r}) \psi_{i}^{\mathrm{A} *}(\mathbf{r})\right],
\end{aligned}
$$

where $\delta D_{i a}$ denotes a matrix element of the density response in the basis of ground-state MOs, and $\left\{\psi_{i}^{\mathrm{A}}, \psi_{a}^{\mathrm{A}}\right\}$ are subsystem-A parts of molecular orbitals

$$
\psi_{p}^{\mathrm{A}}(\mathbf{r})=\sum_{\mu \in \mathrm{A}} C_{\mu p} \phi_{\mu}(\mathbf{r})
$$

The response function $\chi^{\mathrm{S}}\left(\mathbf{r}, \mathbf{r}^{\prime}, \omega\right)$ in Eq. (7) is the usual density-density linear-response function for the non-interacting system,

$$
\chi^{\mathrm{S}}\left(\mathbf{r}, \mathbf{r}^{\prime}, \omega\right)=\sum_{i, a}\left[\frac{\psi_{i}^{*}(\mathbf{r}) \psi_{a}(\mathbf{r}) \psi_{i}\left(\mathbf{r}^{\prime}\right) \psi_{a}^{*}\left(\mathbf{r}^{\prime}\right)}{\omega-\left(\epsilon_{a}-\epsilon_{i}\right)}-\frac{\psi_{i}(\mathbf{r}) \psi_{a}^{*}(\mathbf{r}) \psi_{i}^{*}\left(\mathbf{r}^{\prime}\right) \psi_{a}\left(\mathbf{r}^{\prime}\right)}{\omega+\left(\epsilon_{a}-\epsilon_{i}\right)}\right]
$$

with $\left\{\epsilon_{i}, \epsilon_{a}\right\}$ corresponding to the canonical ground-state MO energies.

Following the same algebraic manipulations as in conventional linear-response TDDFT, ${ }^{43}$ we obtain the response equation for TD-EMFT in the ground-state MO basis,

$$
\left[\left(\begin{array}{cc}
\mathbf{A} & \mathbf{B} \\
\mathbf{B}^{*} & \mathbf{A}^{*}
\end{array}\right)-\omega\left(\begin{array}{rr}
\mathbf{1} & \mathbf{0} \\
\mathbf{0} & -\mathbf{1}
\end{array}\right)\right]\left(\begin{array}{l}
\mathbf{X} \\
\mathbf{Y}
\end{array}\right)=-\left(\begin{array}{c}
\delta \mathbf{v}_{\mathrm{ext}} \\
\delta \mathbf{v}_{\mathrm{ext}}^{\dagger}
\end{array}\right)
$$

Here, $\mathbf{X}$ and $\mathbf{Y}$ correspond to the virtual-occupied and occupied-virtual blocks of the density response matrix,

$$
X_{a i}=\delta D_{a i}, \quad Y_{a i}=\delta D_{i a}
$$

and $\delta \mathbf{v}_{\text {ext }}$ is the external perturbation matrix in the MO basis. The matrices $\mathbf{A}$ and $\mathbf{B}$ 
are defined as

$$
\begin{aligned}
A_{a i, b j}= & \delta_{i j} \delta_{a b}\left(\varepsilon_{a}-\varepsilon_{i}\right)+(a i \mid b j)+\left(a i\left|f_{\mathrm{xc}}^{(2)}\left(\mathbf{r}, \mathbf{r}^{\prime}\right)\right| b j\right) \\
& +\left(a^{\mathrm{A}} i^{\mathrm{A}}\left|f_{\mathrm{xc}, \mathrm{AA}}^{(1)}\left(\mathbf{r}, \mathbf{r}^{\prime}\right)\right| b^{\mathrm{A}} j^{\mathrm{A}}\right)-\left(a^{\mathrm{A}} i^{\mathrm{A}}\left|f_{\mathrm{xc}, \mathrm{AA}}^{(2)}\left(\mathbf{r}, \mathbf{r}^{\prime}\right)\right| b^{\mathrm{A}} j^{\mathrm{A}}\right), \\
B_{a i, b j}= & (a i \mid j b)+\left(a i\left|f_{\mathrm{xc}}^{(2)}\left(\mathbf{r}, \mathbf{r}^{\prime}\right)\right| j b\right) \\
& +\left(a^{\mathrm{A}} i^{\mathrm{A}}\left|f_{\mathrm{xc}, \mathrm{AA}}^{(1)}\left(\mathbf{r}, \mathbf{r}^{\prime}\right)\right| j^{\mathrm{A}} b^{\mathrm{A}}\right)-\left(a^{\mathrm{A}} i^{\mathrm{A}}\left|f_{\mathrm{xc}, \mathrm{AA}}^{(2)}\left(\mathbf{r}, \mathbf{r}^{\prime}\right)\right| j^{\mathrm{A}} b^{\mathrm{A}}\right),
\end{aligned}
$$

where $\left\{a^{\mathrm{A}}, i^{\mathrm{A}}\right\}$ are the simplified notation for $\left\{\psi_{a}^{\mathrm{A}}(\mathbf{r}), \psi_{i}^{\mathrm{A}}(\mathbf{r})\right\}$ defined in Eq. (13). (ai $\left.\mid b j\right)$ and $\left(a i\left|f_{\mathrm{xc}}\left(\mathbf{r}, \mathbf{r}^{\prime}\right)\right| b j\right)$ correspond to the two-electron repulsion integrals and the xc integrals, respectively,

$$
\begin{aligned}
(p q \mid r s) & =\int d \mathbf{r} d \mathbf{r}^{\prime} \psi_{p}^{*}(\mathbf{r}) \psi_{q}(\mathbf{r}) \frac{1}{\left|\mathbf{r}-\mathbf{r}^{\prime}\right|} \psi_{r}^{*}\left(\mathbf{r}^{\prime}\right) \psi_{s}\left(\mathbf{r}^{\prime}\right) \\
\left(p q\left|f_{\mathrm{xc}}\right| r s\right) & =\int d \mathbf{r} d \mathbf{r}^{\prime} \psi_{p}^{*}(\mathbf{r}) \psi_{q}(\mathbf{r}) f_{\mathrm{xc}}\left(\mathbf{r}, \mathbf{r}^{\prime}\right) \psi_{r}^{*}\left(\mathbf{r}^{\prime}\right) \psi_{s}\left(\mathbf{r}^{\prime}\right)
\end{aligned}
$$

Excitation energies are determined as the poles of the response function (i.e. left-handside of Eq. (15)), leading to the TD-EMFT eigenvalue equation,

$$
\left(\begin{array}{cc}
\mathbf{A} & \mathbf{B} \\
\mathbf{B}^{*} & \mathbf{A}^{*}
\end{array}\right)\left(\begin{array}{l}
\mathbf{X} \\
\mathbf{Y}
\end{array}\right)=\omega\left(\begin{array}{rr}
\mathbf{1} & \mathbf{0} \\
\mathbf{0} & -\mathbf{1}
\end{array}\right)\left(\begin{array}{l}
\mathbf{X} \\
\mathbf{Y}
\end{array}\right)
$$

with the eigenvalue $\omega$ corresponding to the excitation energy and the eigenvector $(\mathbf{X}, \mathbf{Y})$ corresponding to the transition density.

Note that the expressions for the xc kernals in Eqs. (9) and (10) remain unchanged for cases in which the low-level theory corresponds to FCDFT, except that the MO energies and coefficients are obtained from the minimization of the energy functional in Eq. (6). This follows from the fact that the Fock-matrix corrections in Eq. (4) involve at most one-body interactions.

TD-EMFT offers potential advantage over conventional linear-response TDDFT in terms of the computational efficiency. Assuming the low-level theory is negligible in cost with respect to the high-level theory, the cost of TD-EMFT is comparable to the 
cost of the TDDFT calculation on subsystem A only, while TD-EMFT includes the environmental response to the excitation. Unlike other embedding approaches such as ONIOM, TD-EMFT allows for straightforward implementation and calculation of excited-state gradients and transition properties such as the non-adiabatic couplings. Furthermore, inherited from its parent ground-state method, TD-EMFT avoids the need to specify the number of electrons or spin state for each subsystem, allowing for the description of charge transfer and particle-number fluctuations between subsystems.

Despite these advantages, several limitations of TD-EMFT are worth noting. In particular, TD-EMFT inherits all of the normal shortcomings of linear-response TDDFT, including poor description of double-excitations due to adiabatic approximation ${ }^{2}$ and poor description of Rydberg or long-ranged charge-transfer excitations due to incorrect long-range behavior in the xc kernels. ${ }^{4}$ Furthermore, since the last two terms in Eq. (8) only involve integration over fluctuations in the subsystem A density, this expression for the one-particle perturbation potential confines the high-level description of the response to subsystem A; the expression in Eq. (8) is thus akin to the EX0 implementation of ground-state EMFT for hybrid functionals, ${ }^{37,38}$ for which the exact exchange contribution of the hybrid functional is included only within the subsystem A block of the Fock matrix. Given these approximations, it is expected that delocalized excitations will be challenging to accurately describe using TD-EMFT, such as the example of the $\pi \rightarrow \pi^{*}$ excitation in decapentaene that is presented below.

\section{Computational Details}

Unless otherwise indicated, all results are obtained using the entos molecular simulation package. ${ }^{44}$ Benchmark calculations address the localized $n \rightarrow \pi^{*}$ excitation in decanoic acid, the localized $\pi \rightarrow \pi^{*}$ excitations in nonylbenzene, and the delocalized $\pi \rightarrow \pi^{*}$ excitation in decapentaene. We also include applications of TD-EMFT to the lowest Qband excitations in chlorophyll a, the study of solvatochromic shifts for para-nitroaniline (pNA) in water, and the sulfur K-edge X-ray absorption spectra (XAS) for the amino 
acid cysteine and its oxidized dimer cystine. We analyze the transition properties of these molecules including the excitation energy, the oscillator strength, and the weight of the corresponding orbital transitions, defined as

$$
w_{i a}=\frac{X_{i a}^{2}-Y_{i a}^{2}}{\langle\mathbf{X}+\mathbf{Y} \mid \mathbf{X}-\mathbf{Y}\rangle},
$$

where $X_{i a}, Y_{i a}$ are the transition density matrix elements defined in Eq. (16).

All TD-EMFT calculations reported here describe DFT-in-DFT or DFT-in-FCDFT embedding, in which the high-level theory corresponds to DFT and the low-level theory corresponds to either DFT or FCDFT. The notation "PBE-in-LDA" (or "B3LYP-inLDA") indicates that the $\mathrm{PBE}^{45}$ (or B3LYP ${ }^{46,47}$ ) functional is employed for subsystem A, and LDA is used for subsystem B; likewise, "PBE-in-FCLDA" and "B3LYPin-FCLDA" indicate the use of FCLDA for subsystem B. Table 1 lists the functionals and AO basis sets describing the high- and low-level theories for TD-EMFT calculations on the systems studied in this work. The notation "same-basis" corresponds to the situation where subsystems A and B employ the same AO basis set; and the notation "mixed-basis" indicates that the subsystems employ different AO basis sets, with subsystem B using the minimal STO-3G basis set.

Table 1. List of the high- and low-level functionals and AO basis sets used in TD-EMFT for the systems studied in this work.

\begin{tabular}{c|cccccc}
\hline \hline \multirow{2}{*}{ System } & \multicolumn{2}{|c}{ Functional } & & \multicolumn{2}{c}{ AO basis } \\
\cline { 2 - 3 } \cline { 6 - 7 } & High-level & Low-level & & High-level & Low-level \\
\hline \hline Decanoic acid & PBE & LDA & & $6-31 G^{*}$ & STO-3G \\
Nonylbenzene & B3LYP & LDA & & $6-311 G^{* *}$ & STO-3G \\
Chlorophyll a & B3LYP & LDA & & $6-31 G^{*}$ & STO-3G \\
pNA in water & B3LYP & LDA & & $6-31 G^{*}$ & STO-3G \\
Decapentaene & B3LYP & LDA & & $6-311 G^{* *}$ & STO-3G \\
\hline \hline
\end{tabular}

Density fitting is employed in all calculations for the evaluation of the Coulomb and exact exchange integrals. ${ }^{48}$ The Ahlrichs Coulomb Fitting basis ${ }^{49,50}$ is used for the 
PBE-in-LDA and PBE-in-FCLDA embedding calculations, and the cc-pVDZ/JKFIT basis $^{51}$ is used for the B3LYP-in-LDA and B3LYP-in-FCLDA embedding calculations. For cases with the same-basis implementation, the full density-fitting basis is used for both subsystems A and B; for cases with the mixed-basis implementation, the full density-fitting basis is used for subsystem A, and only the s-type functions of the corresponding density-fitting basis is used for subsystem B. Both the AO and densityfitting functions are implemented as spherical Gaussians.

For EMFT and TD-EMFT calculations that employ the B3LYP functional for the high-level mean-field theory, the EX0 implementation is used, for which the exact exchange contribution of the hybrid functional is included only within the subsystem A block of the Fock matrix. ${ }^{37,38}$ Block-orthogonalized (BO) partitioning is employed for calculations with the same-basis B3LYP-in-LDA embedding to avoid unphysical collapse of the ground-state EMFT solutions. ${ }^{38}$

For calculations employing FCLDA, we use the full Fock-matrix corrections for systems composed of only carbons and hydrogens, with the FCLDA parameters provided in Ref. 39; we use the diagonal-only Fock-matrix corrections for systems containing elements other than carbon and hydrogen, with the parameters provided in Ref. 41.

For comparison, we also provide results obtained using conventional linear-response TDDFT for the full system. Additionally, we provide results in some cases using TDDFT with vacuum embedding, in which subsystem A is terminated with hydrogen link-atoms and treated at the high-level of theory. The terminal hydrogen link-atoms are positioned according to the default parametrization scheme for ONIOM in Gaussian 09. ${ }^{52}$ All geometries are optimized at the B3LYP/6-31G* level, with the Cartesian coordinates as well as the the specification of EMFT partitioning of subsystems provided in the Supporting Information. 


\section{Results and Discussion}

\subsection{Localized $n \rightarrow \pi^{*}$ excitation in decanoic acid}

We first evaluate the performance of TD-EMFT for localized excitations, focusing on the $n \rightarrow \pi^{*}$ excitation in decanoic acid. Figure 1 shows the subsystem-size dependence of the TD-EMFT results in terms of the embedding error in the excitation energies (Figure 1a), the oscillator strength (Figure 1b), and the weight of $n \rightarrow \pi^{*}$ transition (Figure 1c). Three implementations of TD-EMFT are considered: same-basis PBEin-LDA, mixed-basis PBE, and mixed-basis PBE-in-LDA. The embedding errors in Figure 1a plot the difference between the TD-EMFT results and TDDFT performed on the full system at the PBE/6-31G* level.
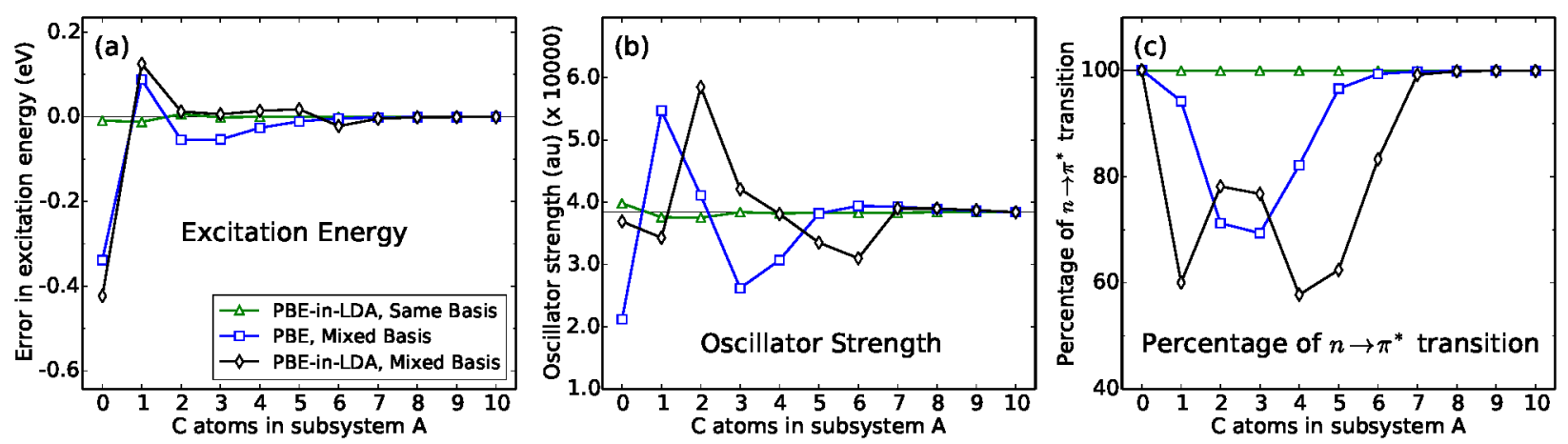

Figure 1. TD-EMFT results for the $n \rightarrow \pi^{*}$ excitation of decanoic acid with respect to the size of subsystem A: (a) embedding errors in the excitation energy, (b) oscillator strength, and (c) weight of the $n \rightarrow \pi^{*}$ orbital transition. The $x$-axes indicate the number of carbon atoms from the alkane chain plus the carbon atom from the carboxyl group.

For the same-basis PBE-in-LDA embedding (green curves in Figure 1), TD-EMFT accurately describes the $n \rightarrow \pi^{*}$ excitation at all subsystem sizes, reproducing the excitation energy, oscillator strength, and percentage of $n \rightarrow \pi^{*}$ transition from those obtained using TDDFT at the PBE/6-31G* level. However, the mixed-basis TD-EMFT results are substantially worse, with both mixed-basis PBE and mixed-basis PBE-inLDA embedding exhibiting slow convergence with respect to subsystem size for the calculation of the oscillator strength (Figure 1b) and percentage of $n \rightarrow \pi^{*}$ transition (Figure 1c). 


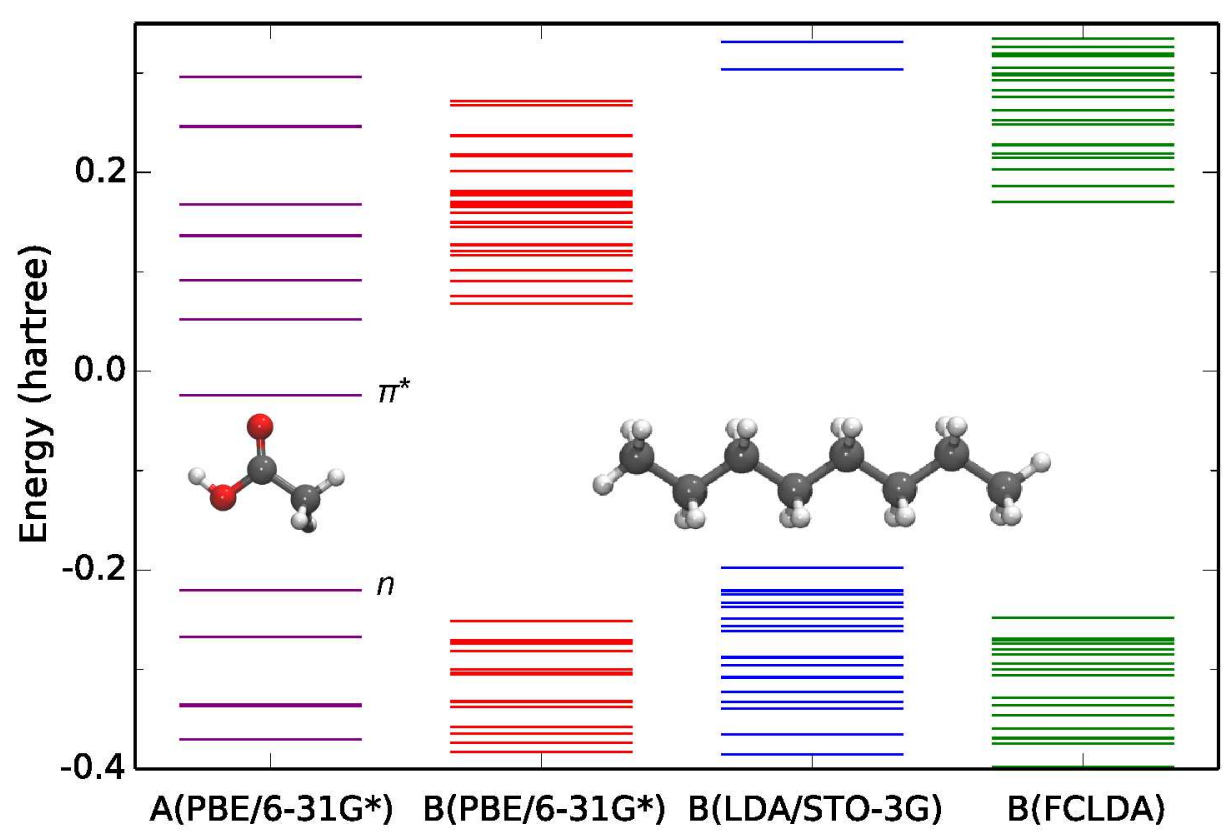

Figure 2. MO energies for closed-shell molecular fragments derived from decanoic acid. Fragment A corresponds to acetic acid and fragment B corresponds to octane. The $x$-axis indicates the fragment and the level of theory used for obtaining the MO energies.

The errors in Figure 1 for the excited-state properties obtained with mixed-basis TD-EMFT are attributed to the mismatch of the MO energies between the levels of theory used to describe subsystems A and B. Figure 2 illustrates this by comparing the MO energies for closed-shell molecular fragments derived from decanoic acid, with fragment $\mathrm{A}$ corresponding to acetic acid and fragment B corresponding to octane. When the same level of theory (PBE/6-31G*) is used to described the two fragments, all of the occupied orbitals of fragment B lie well below the HOMO of fragment A (i.e., the lone-pair orbital) and all of the unoccupied orbitals of fragment B lie well above the LUMO of fragment A (i.e., the $\pi^{*}$ orbital). As a result, the lowest excited state of decanoic acid obtained from TDDFT at the PBE/6-31G* level is dominated by the $n \rightarrow \pi^{*}$ transition from the carboxyl group. However, in a TD-EMFT calculation in which subsystem B is described by the LDA/STO-3G level, the orbitals of subsystem $\mathrm{B}$ are shifted to higher energies such that the HOMO of subsystem B becomes close in energy to the HOMO of subsystem A. This mismatch leads to contamination of the $n \rightarrow \pi^{*}$ excitations obtained from TD-EMFT with mixed-basis embedding, as seen in 
Figure 1b and Figure 1c.
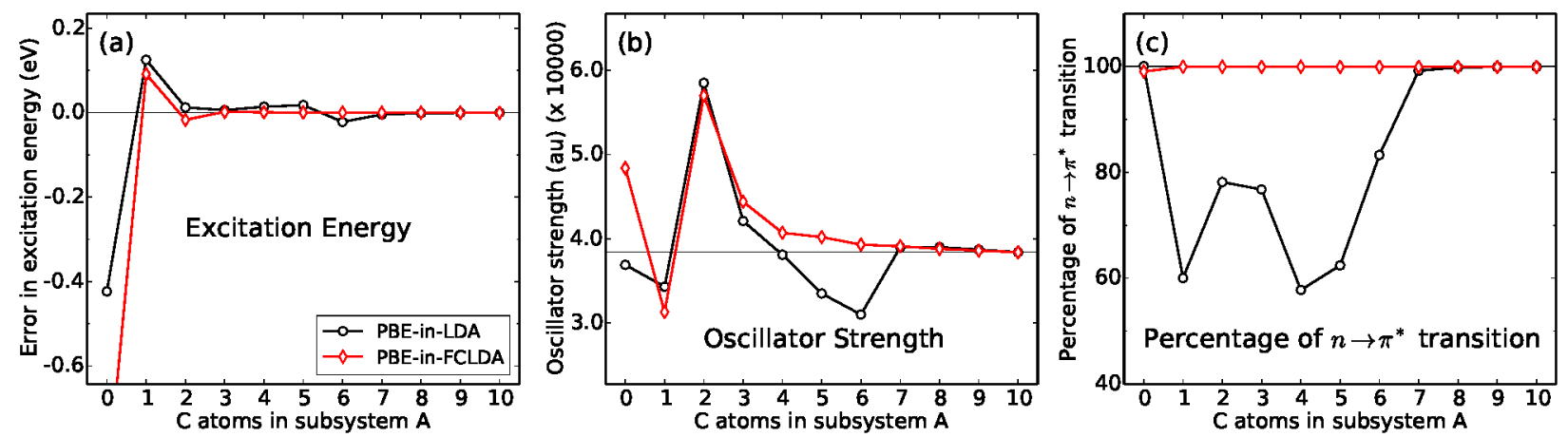

Figure 3. Comparison of TD-EMFT without (black) and with (red) Fock-matrix corrections for the $n \rightarrow \pi^{*}$ excitation of decanoic acid with respect to the subsystem size: (a) errors in the excitation energy, (b) oscillator strength, and (c) weight of $n \rightarrow \pi^{*}$ orbital transition.

As was previously shown for ground-state mixed-basis EMFT calculations, ${ }^{41}$ errors associated with mismatch of the subsystem MO energies can be alleviated using FCDFT. Figure 3 demonstrates that including Fock-matrix corrections in the TDEMFT calculation of the $n \rightarrow \pi^{*}$ excitation of decanoic acid likewise improves the description of the excited state, yielding smaller errors and better convergence in the excitation energy, oscillator strength, and percentage of $n \rightarrow \pi^{*}$ character. The final column in Figure 2 confirms that the improvements with Fock-matrix corrections indeed arise from correction of the molecular orbital mismatch between the subsystems; the molecular orbital energies for fragment B with Fock-matrix corrections are shifted to be in excellent agreement with the $\mathrm{PBE} / 6-31 \mathrm{G}^{*}$ results. These results suggest that employment of FCDFT will alleviate errors in the TD-EMFT description of excitations that primarily arise due to mismatch in valence orbital energies between the high- and low-level methods.

\subsection{Localized $\pi \rightarrow \pi^{*}$ excitations in nonylbenzene, chloro- phyll a, and solvated dye molecule}

We now consider three examples involving localized $\pi \rightarrow \pi^{*}$ excitations in various molecular environments. The first system considered is nonylbenzene, for which the 
two lowest excited states are both dominated by $\pi \rightarrow \pi^{*}$ transitions from the phenyl group (Figure 4). As shown in Figure 4 , the ${ }^{1} B_{2 u}$-like excited state is dominated by the $\mathrm{HOMO} \rightarrow$ LUMO and $\mathrm{HOMO}-1 \rightarrow \mathrm{LUMO}+1$ orbital pair excitations, whereas the ${ }^{1} B_{1 u}$-like excited state is characterized by the $\mathrm{HOMO} \rightarrow \mathrm{LUMO}+1$ and $\mathrm{HOMO}-1 \rightarrow$ LUMO orbital pair excitations.
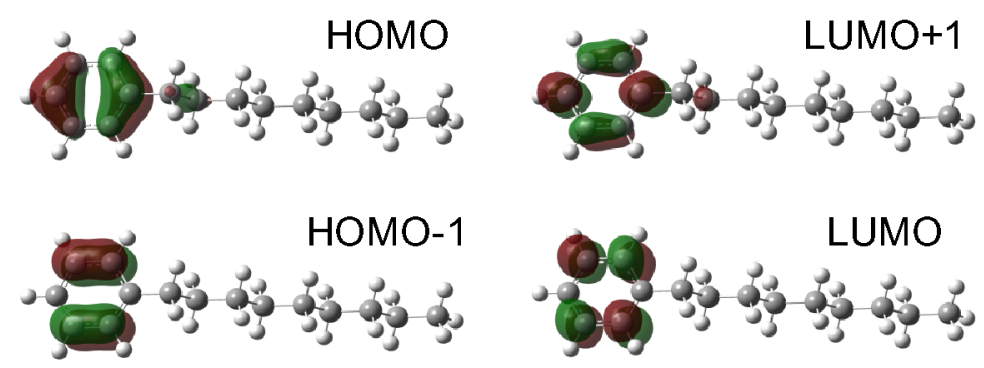

Figure 4. Frontier MOs involved in the ${ }^{1} \mathrm{~B}_{1 \mathrm{u}}$-like and ${ }^{1} \mathrm{~B}_{2 \mathrm{u}}$-like excited states of nonylbenzene.

We investigate the performance of TD-EMFT for these excitations using mixed-basis B3LYP-in-LDA embedding. Figure 5 shows the subsystem-size dependence of the TDEMFT results both without (black) and with (red) Fock-matrix corrections. For both excited states, TD-EMFT without Fock-matrix corrections gives slow convergence of the transition properties (i.e. excitation energy, oscillator strength, and the transition character), which is again attributed to mismatch of the subsystem MO energies due to use of the mixed-basis set. As before, the use of Fock-matrix corrections substantially improves the convergence of all three transition properties with respect to the subsystem size. 

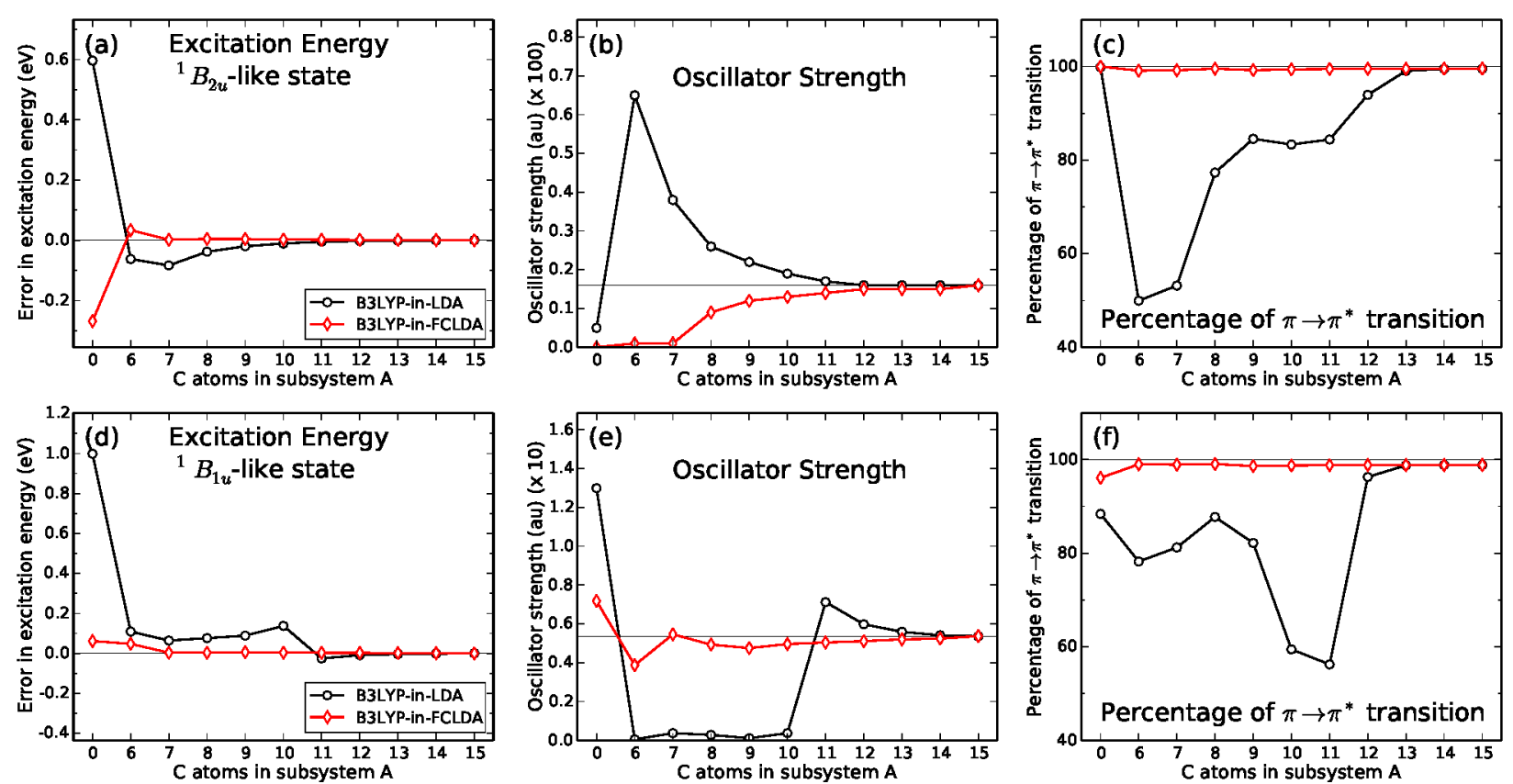

Figure 5. TD-EMFT results for the ${ }^{1} B_{2 u}$-like (a-c) and ${ }^{1} B_{1 u}$-like (d-f) excited states for nonylbenzene with respect to the subsystem size: (a, d) embedding errors in the excitation energy, (b, e) oscillator strength, and (c, f) percentage of $\pi \rightarrow \pi^{*}$ orbital transition. The $x$-axes indicate the number of carbon atoms from the alkane chain plus the number of carbon atoms from the phenyl group.

We next consider the localized $\pi \rightarrow \pi^{*}$ excitations in chlorophyll a (Figure 6), one of the essential pigments for light absorption in natural photosynthesis. Previous theoretical studies on this system ${ }^{53-60}$ have focused on the structural and environmental effects on its absorption/emission properties, employing semi-empirical methods, ${ }^{56-58}$ correlated wavefunction methods for truncated model structures, ${ }^{54}$ TDDFT for lowenergy absorption spectra, ${ }^{53,55,59}$ and the subsystem TDDFT approach ${ }^{16,60-63}$ based on the frozen density embedding. 
Figure 6. Geometry and schematic representation of the subsystem partitioning for chlorophyll a.The atoms associated with subsystem $A$ are shown as solid balls, and the atoms in subsystem $B$ are shown as translucent.

We consider the $\mathrm{Q}_{\mathrm{x}}$ - and $\mathrm{Q}_{\mathrm{y}}$-band excitations of chlorophyll a, both of which correspond to $\pi \rightarrow \pi^{*}$ excitations within the magnesium-binding moiety of the molecule. Figure 6 illustrates the subsystem partitioning used for the TD-EMFT calculations reported here, with the atoms of subsystem A highlighted in red. Table 2 shows the TDEMFT results obtained using the mixed-basis B3LYP-in-LDA and B3LYP-in-FCLDA embedding. The TD-EMFT results are compared with TDDFT results obtained at the B3LYP/6-31G* and LDA/STO-3G levels of theory, as well as results obtained using vacuum embedding.

As seen in Table 2, TDDFT with LDA/STO-3G gives large errors relative to TDDFT with $\mathrm{B} 3 \mathrm{LYP} / 6-31 \mathrm{G}^{*}$ for the various transition properties of the two excited states. TD-EMFT without Fock-matrix corrections improves the excitation energies, but gives 
qualitatively inaccurate description of the excitation character in terms of the oscillator strength and orbital transition weights, due to MO-energy mismatch arising from the mixed basis set. Employment of Fock-matrix corrections greatly improves the description of both excited states, leading to excellent agreement with TDDFT at the B3LYP $/ 6-31 \mathrm{G}^{*}$ level in terms of the excitation energies, oscillator strength, and orbital transition characters. However, the success of vacuum embedding for this application indicates that it is not a challenging case for embedding methods, since complete neglect of polarization in subsystem B still leads to good agreement with TDDFT calculations performed over the full system; more challenging applications related to substantial solvent effects and delocalized excitations are explored next.

Table 2. Excitation energies (in $\mathrm{eV}$ ), oscillator strengths (in au), and weight of dominant orbital transitions for the $\mathrm{Q}_{\mathrm{x}}$ - and $\mathrm{Q}_{\mathrm{y}}$-band excitations in chlorophyll a, obtained using TD-EMFT with mixed-basis B3LYP-inLDA and B3LYP-in-FCLDA embedding, TDDFT at the B3LYP/6-31G*and LDA/STO-3G levels of theory, as well as TDDFT with vacuum embedding.

\begin{tabular}{|c|c|c|c|c|c|}
\hline & \multicolumn{2}{|c|}{ TDDFT } & \multicolumn{2}{|c|}{ Mixed-basis TD-EMFT } & \multirow{2}{*}{$\begin{array}{c}\text { Vac. Embed. } \\
\text { B3LYP } / 6-31 G^{*}\end{array}$} \\
\hline & B3LYP/6-31G* & LDA/STO-3G & B3LYP-in-LDA & B3LYP-in-FCLDA & \\
\hline \multicolumn{6}{|c|}{$\mathrm{Q}_{\mathrm{y}}$-band excitation } \\
\hline Excitation energy $(\mathrm{eV})$ & 2.14 & 2.45 & 2.14 & 2.15 & 2.18 \\
\hline Oscillator strength $(\mathrm{au})$ & 0.228 & 0.123 & 0.162 & 0.221 & 0.201 \\
\hline \multicolumn{6}{|c|}{ Orbital transition weight (\%) } \\
\hline HOMO-1 $\rightarrow$ LUMO +1 & 14.1 & 38.1 & 9.6 & 14.4 & 16.4 \\
\hline HOMO $\rightarrow$ LUMO & 85.5 & 52.4 & 58.1 & 85.2 & 82.2 \\
\hline \multicolumn{6}{|c|}{$\mathrm{Q}_{\mathrm{x}}$-band excitation } \\
\hline Excitation energy (eV) & 2.31 & 2.37 & 2.33 & 2.34 & 2.30 \\
\hline Oscillator strength (au) & 0.025 & 0.042 & 0.015 & 0.018 & 0.035 \\
\hline \multicolumn{6}{|c|}{ Orbital transition weight (\%) } \\
\hline HOMO-1 $\rightarrow$ LUMO & 70.9 & 60.7 & 64.4 & 68.2 & 74.0 \\
\hline $\mathrm{HOMO} \rightarrow \mathrm{LUMO}+1$ & 27.4 & 16.9 & 21.9 & 30.1 & 23.9 \\
\hline
\end{tabular}


Figure 7. Geometry and schematic representation of the subsystem partitioning for para-nitroaniline in $\left(\mathrm{H}_{2} \mathrm{O}\right)_{18}$ cluster. Solid balls represent atoms in the pNA molecule, which corresponds to subsystem $A$; the surrounding 18 water molecules correspond to subsystem B and are shown as translucent.

As a third example, we consider the solvatochromic shift associated with the localized $\pi \rightarrow \pi^{*}$ charge-transfer (CT) excitation of para-nitroaniline (pNA). Previous theoretical studies for this system have employed TDDFT with cluster models for the solvation, ${ }^{64,65}$ continuum solvation models, ${ }^{66} \mathrm{QM} / \mathrm{MM},{ }^{67-70}$ and frozen-density embedding. ${ }^{71}$ Here, we employ TD-EMFT, using a cluster model composed of the pNA molecule and 18 explicit water molecules representing the first solvation shell (Figure 7). Table 3 presents the TD-EMFT results obtained using the mixed-basis B3LYPin-LDA and B3LYP-in-FCLDA embedding, with subsystem A corresponding to the pNA molecule. For comparison, we also include results obtained using TDDFT at the B3LYP/6-31G* and LDA/STO-3G levels of theory applied to the full cluster. The solvatochromic shifts are computed relative to the gas-phase results, which are obtained using TDDFT at the B3LYP/6-31G* level for the bare pNA molecule.

As in the previous example, TDDFT at the LDA/STO-3G level gives substantial errors, overestimating the solvatochromic shift by $\sim 0.4 \mathrm{eV}$ and producing too low 
oscillator strength and the weight of the $\pi \rightarrow \pi^{*}$ transition. TD-EMFT without Fockmatrix corrections improves the accuracy in the solvatochromic shift, reducing the error to $\sim 0.06 \mathrm{eV}$ with respect to TDDFT at the B3LYP/6-31G* level, but it gives qualitatively incorrect description for the transition character, with reduced oscillator strength (0.098) and the weight of the $\pi \rightarrow \pi^{*}$ transition (48.7\%), due to MO-energy mismatch arising from the mixed basis set. Table 3 shows that TD-EMFT with Fockmatrix corrections greatly improved the description for all three transition properties, leading to much better agreement with TDDFT at the B3LYP/6-31G* level.

Table 3. Solvatochromic shifts (in eV), oscillator strengths (in atomic units), and weight of the lowest $\pi \rightarrow \pi^{*}$ orbital transition of pNA in $\left(\mathrm{H}_{2} \mathrm{O}\right)_{18}$ cluster, obtained using TD-EMFT with mixedbasis B3LYP-in-LDA and B3LYP-in-FCLDA embedding, and TDDFT at the B3LYP/6-31G* and LDA/STO-3G levels of theory. The solvatochromic shifts are computed relative to the gas-phase results, which are obtained using TDDFT with B3LYP/6-31G* for the bare pNA molecule. Numbers in the parenthesis correspond to errors in the solvatochromic shift with respect to TDDFT at the B3LYP/6-31G* level.

\begin{tabular}{lccccc}
\hline \hline & \multicolumn{2}{c}{ TDDFT } & & \multicolumn{2}{c}{ TD-EMFT } \\
\cline { 2 - 3 } \cline { 5 - 6 } & B3LYP/6-31G** & LDA/STO-3G & & B3LYP-in-LDA & B3LYP-in-FCLDA \\
\hline Solvatochromic shift $(\mathrm{eV})$ & -0.68 & $-0.28(0.40)$ & & $-0.74(-0.06)$ & $-\mathbf{0 . 7 5}(-\mathbf{0 . 0 7})$ \\
Oscillator strength $(\mathrm{au})$ & 0.487 & 0.140 & & 0.098 & $\mathbf{0 . 4 4 5}$ \\
Weight of $\pi \rightarrow \pi^{*}(\%)$ & 84.4 & 48.7 & & 60.0 & $\mathbf{9 5 . 6}$ \\
\hline \hline
\end{tabular}

\subsection{Delocalized $\pi \rightarrow \pi^{*}$ excitation in a polyene}

Having demonstrated TD-EMFT for the localized excitations, we now consider the challenging case of a delocalized $\pi \rightarrow \pi^{*}$ excitation in decapentaene. This system is demanding for embedding approaches because the excitation occurs over the entire molecule and inconsistent treatment of the subsystems can lead to large errors in the description of the excitation. 

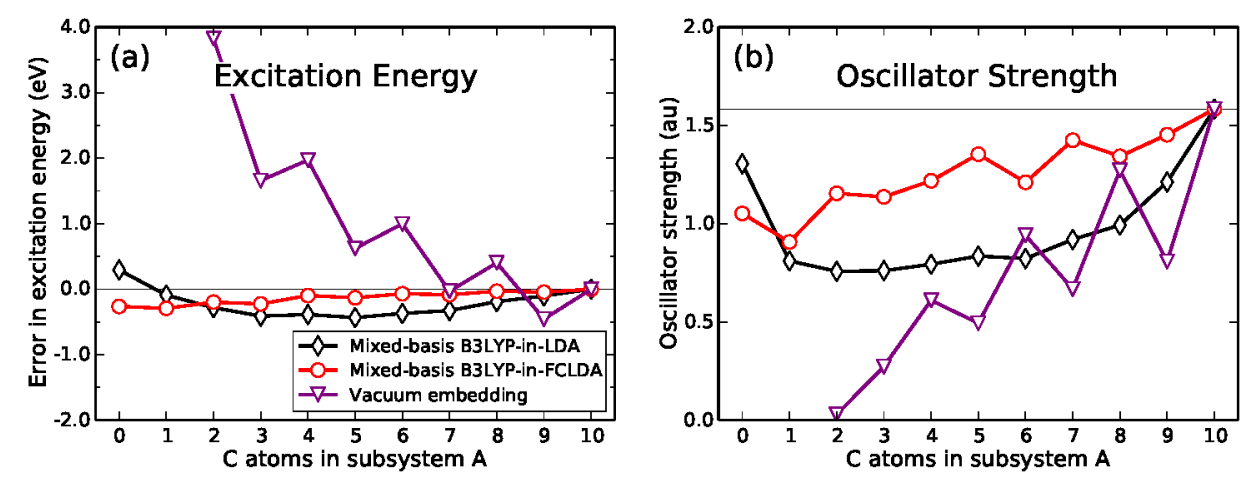

Figure 8. TD-EMFT results for the $\pi \rightarrow \pi^{*}$ excitation of decapentaene with respect to the subsystem size: (a) embedding errors in the excitation energy and (b) the oscillator strengths, obtained using mixed-basis B3LYP-in-LDA, mixedbasis B3LYP-in-FCLDA and vacuum embedding.

Figure 8 shows the subsystem-size dependence of the TD-EMFT results using mixedbasis B3LYP-in-LDA (black curves), mixed-basis B3LYP-in-FCLDA embedding (red curves), and vacuum embedding (purple curves). It is clear from Figure 8 that vacuum embedding fails to give even qualitative description for the $\pi \rightarrow \pi^{*}$ excitation of the whole system, producing significant errors in both the excitation energy and oscillator strength at all subsystem sizes. For small subsystem sizes (with less than 5 carbons in subsystem $\mathrm{A}$ ), the errors in the excitation energy are greater than $1 \mathrm{eV}$. Moreover, the errors oscillate substantially due to the change in the conjugation pattern across subsystem A. TD-EMFT with mixed-basis B3LYP-in-LDA embedding improves the description of the $\pi \rightarrow \pi^{*}$ excitation, reducing the embedding errors in the excitation energy to within $0.5 \mathrm{eV}$. However, these errors are still larger than those seen for the localized excitations, as discussed in previous examples.

To again address the errors caused by use of the mixed basis set, we employ Fockmatrix corrections to TD-EMFT with the mixed-basis B3LYP-in-LDA embedding. As seen in Figure 8, the use of Fock-matrix corrections greatly reduces errors in both the excitation energy and oscillator strength, and shows better convergence with respect to the subsystem size. 


\subsection{Sulfur K-edge X-ray absorption spectra for cysteine and cystine}

Near-edge X-ray absorption spectroscopy (XAS) probes excitations of core electrons to the unoccupied valence orbitals and is a powerful experimental technique to probe information about the chemical composition and the electronic structure in biological systems, ${ }^{72-75}$ surfaces, ${ }^{76}$ thin films, ${ }^{77}$ and small molecules in the gas phase. ${ }^{78}$ Theory can play a crucial role in aiding the interpretation of the experimental spectra. ${ }^{74}$ Several theoretical approaches have been developed to simulate XAS spectra, which includes methods based on TDDFT, ${ }^{79-82} \Delta$ SCF-based approaches, ${ }^{83,84}$ the excited-state core-hole $(\mathrm{XCH})$ method, ${ }^{85}$ the multiple scattering $\chi_{\alpha}$ methods, ${ }^{86}$ the equation-ofmotion or linear-response coupled cluster approaches, ${ }^{87}$ and the second-order algebraic diagrammatic construction scheme (ADC). ${ }^{88,89}$ Most of these methods require DFT or more expensive calculations on the full system, which can become less feasible for large systems. TD-EMFT is potentially useful for simulating XAS spectra because of the localized nature of the corresponding excitations, which the previously discussed benchmark results show to be accurately described.
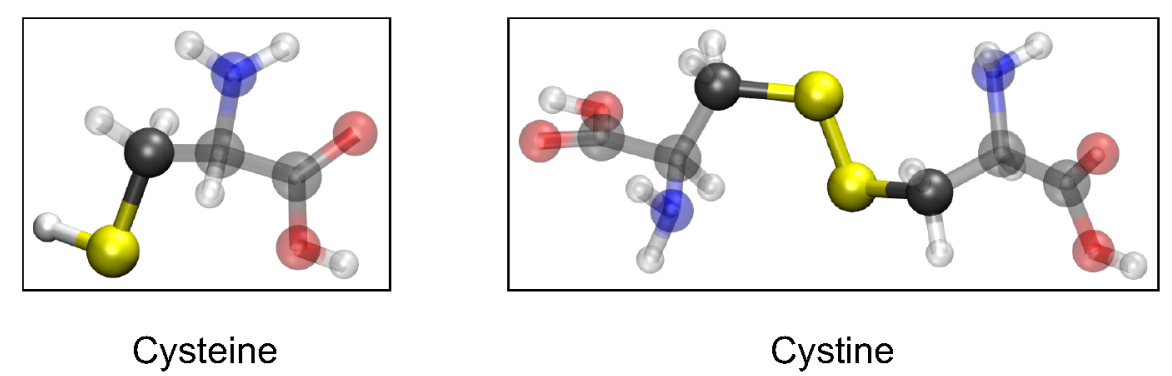

Figure 9. Geometry and schematic representation of the EMFT subsystem partitioning for cysteine and cystine. The solid balls represent atoms in subsystem $\mathrm{A}$; the remaining atoms correspond to subsystem B and are shown as translucent.

We calculate the sulfur K-edge XAS spectra, which enables the in vivo detection and the quantification of thiols and disulfides in biological systems. ${ }^{72,90}$ We apply both TDDFT and TD-EMFT to the amino acid cysteine and its dimer cystine. For TDDFT calculations, we use the $\mathrm{BH}^{0.71} \mathrm{LYP}$ functional, which corresponds to a modified BHLYP 
functional with $71 \%$ of HF exchange, as it was shown to give smaller errors than standard hybrid functionals for core-excitations for second-row elements; ${ }^{91}$ the $6-311++\mathrm{G}^{* *}$ basis set is used to provide an adequate description of both core and unoccupied orbitals. For TD-EMFT calculations, the sulfur atoms and their nearest-neighbor atoms are treated at the $\mathrm{BH}^{0.71} \mathrm{LYP} / 6-311++\mathrm{G}^{* *}$ level, as indicated by the highlighted atoms in Figure 9; the remaining atoms are treated at the LDA/STO-3G level.

Figure 10 shows the simulated spectra for the two molecules. Relativistic effects on the sulfur 1s orbital were included by applying a correction of $7.4 \mathrm{eV}$ to all calculated excitation energies, and the computed spectra were convolved with a Gaussian function with full-with-at-half-maximum (FWHM) of $0.5 \mathrm{eV}$. All calculated spectra were universally shifted by $0.9 \mathrm{eV}$ to higher energy to match the position of the main experimental peak of cysteine. Table 4 reports the dominant excitations to the XAS spectra in terms of the unshifted excitation energies and oscillator strengths. For comparison, we also show in Figure 10 and Table 4 the experimental spectra and main peak positions of cysteine and cystine measured in their solid phase.

It is clear from Figure 10 that TD-EMFT gives spectra almost identical to TDDFT, reproducing both the main absorption features and the relative peak positions. Furthermore, both the TDDFT and TD-EMFT simulated spectra agree well with experiment. The experimental spectra of both cysteine (Figure 10a) and cystine (Figure 10b) exhibit intense absorptions in the low-energy regime, and a very broad low-intensity band in the high-energy region. For cysteine (Figure 10a), the low-energy regime exhibits only one intense peak, corresponding to excitations from the sulfur 1s orbital to the antibonding orbitals dominated by $\sigma_{\mathrm{S}-\mathrm{H}}^{*}$ and $\sigma_{\mathrm{S}-\mathrm{C}}^{*}$ character. For cystine (Figure 10b), the low-energy region of the spectrum exhibits two intense peaks, with the fist peak corresponding to excitations from the two sulfur 1s orbitals to the anti-bonding orbital with $\sigma_{\mathrm{S}-\mathrm{S}}^{*}$ character, and the second peak corresponding to excitations to the anti-bonding orbitals dominated by $\sigma_{\text {S-C }}^{*}$ character. For these intense excitations, TD-EMFT produces excitation energies and oscillator strengths that are very similar to full TDDFT (with less than $0.05 \mathrm{eV}$ errors in the excitation energy), as indicated in Table 4. Compared 
to experiment, both TDDFT and TD-EMFT predict the intense peak positions with a uniform error of $\sim 0.9 \mathrm{eV}$, which can be attributed to the self-interaction error of the $\mathrm{BH}^{0.71} \mathrm{LYP}$ functional. For the high-energy regimes of the spectra (excitations above $2475 \mathrm{eV}$ ), the agreement between TD-EMFT, TDDFT, and experiment is also reasonable, despite the strong mixing between excitations to the low-lying valence orbitals and excitations to the delocalized Rydberg orbitals, and larger embedding errors at higher energies. For completeness, we have also performed TDDFT calculations using vacuum embedding (results not shown), which produces a red-shift of more than $0.15 \mathrm{eV}$ for the intense excitations, and much worse predictions for the high-energy excitations as expected due to the stronger effects from subsystem B.

Overall, Figure 10 shows that TD-EMFT quantitatively reproduces the sulfur Kedge XAS spectra obtained from the more expensive TDDFT calculations for cysteine and cystine, and agreement between calculated and experimental spectra suggests that TD-EMFT can be a useful alternative to TDDFT in simulating and interpreting XAS spectra for large systems. 

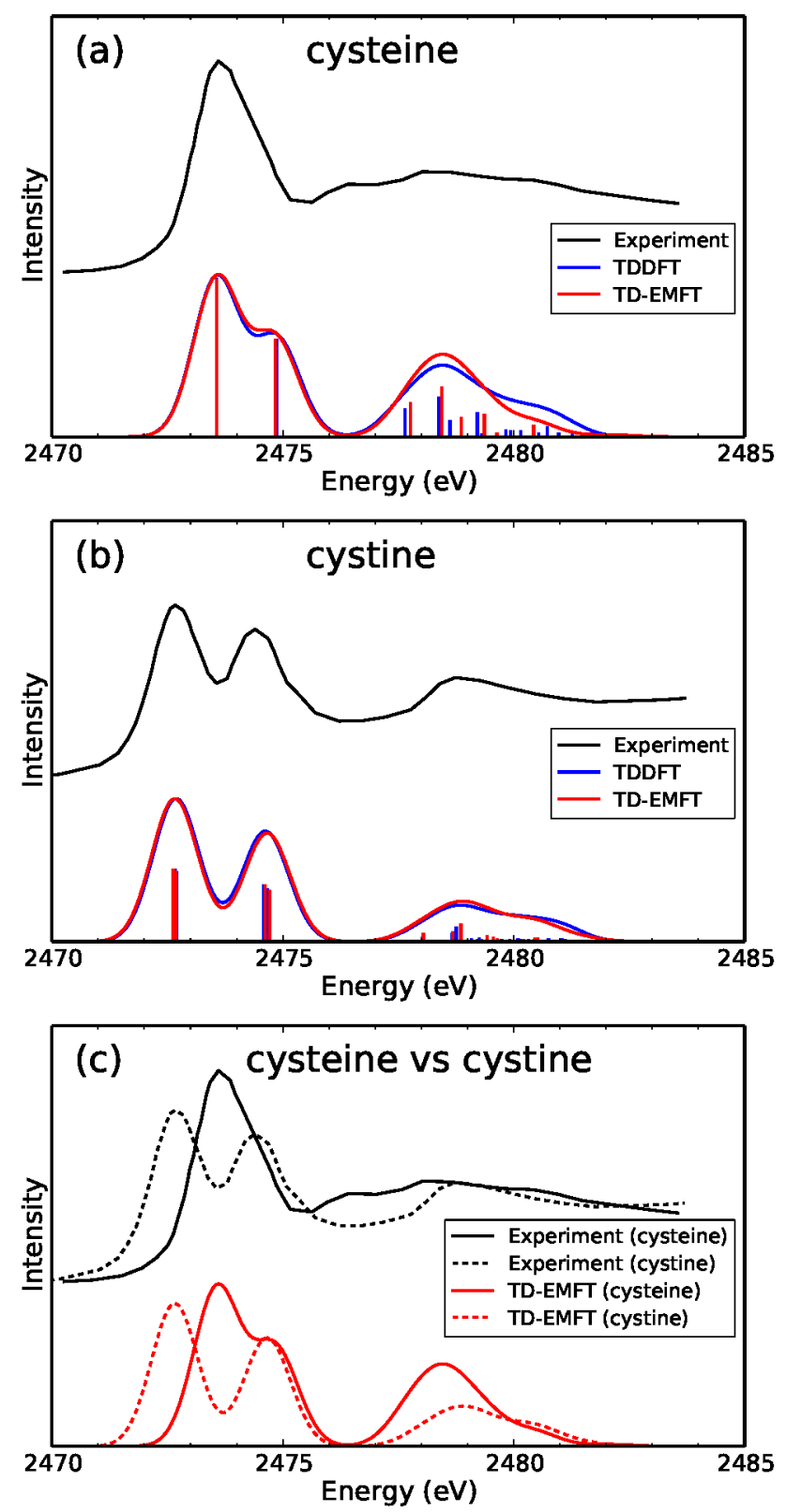

Figure 10. TDDFT (blue), TD-EMFT (red), and experimental (black) sulfur Kedge XAS absorption spectra for: (a) cysteine and (b) cystine. Panel (c) directly compares the spectra of cysteine (solid lines) and cystine (dashed lines) obtained from experiment (black) and TD-EMFT calculations (red). The TDDFT spectra were calculated using a modified B3LYP functional with $71 \%$ of HF exchange (labeled as " $\mathrm{BH}^{0.71} \mathrm{LYP}$ ") and $6-311++\mathrm{G}^{* *}$ basis set. The TD-EMFT spectra were calculated using the mixed-basis $\mathrm{BH}^{0.71} \mathrm{LYP}$-in-LDA embedding with the subsystems partitioning shown in Figure 9. All computed spectra were convoluted with a Gaussian function with full-with-at-half-maximum (FWHM) of $0.5 \mathrm{eV} . \mathrm{A}$ constant shift of $0.9 \mathrm{eV}$ in the excitation energy was applied to all computed spectra to align the main absorption peak of cysteine to the experimental value. The experimental spectra were adapted from Ref. 72. 
Table 4. Computed excitation energies (in eV) and oscillator strengths (in hartree) for the intense excitations corresponding to $\mathrm{S}(1 \mathrm{~s}) \rightarrow \sigma^{*}$ transitions in cysteine and cystine.

\begin{tabular}{|c|c|c|c|c|c|c|}
\hline \multirow{2}{*}{ Molecule } & \multirow{2}{*}{ Excitation } & \multirow{2}{*}{$\begin{array}{c}\text { Exp. }{ }^{a} \\
\text { Energy }\end{array}$} & \multicolumn{2}{|c|}{$\mathrm{TDDFT}^{\mathrm{b}}$} & \multicolumn{2}{|c|}{ TD-EMFT $^{\mathrm{b}}$} \\
\hline & & & Energy & Osc. strength & Energy & Osc. strength \\
\hline \multirow{2}{*}{ cysteine } & $\mathrm{S}(1 \mathrm{~s}) \rightarrow \sigma_{\mathrm{S}-\mathrm{H}}^{*}$ & 2473.6 & 2472.67 & 0.0083 & 2472.67 & 0.0083 \\
\hline & $\mathrm{S}(1 \mathrm{~s}) \rightarrow \sigma_{\mathrm{S}-\mathrm{C}}^{*}$ & 2474.4 & 2473.97 & 0.0051 & 2473.93 & 0.0051 \\
\hline \multirow{4}{*}{ cystine } & \multirow{2}{*}{$\mathrm{S}(1 \mathrm{~s}) \rightarrow \sigma_{\mathrm{S}-\mathrm{S}}^{*}$} & \multirow{2}{*}{2472.7} & 2471.77 & 0.0095 & 2471.73 & 0.0095 \\
\hline & & & 2471.81 & 0.0092 & 2471.79 & 0.0092 \\
\hline & \multirow{2}{*}{$\mathrm{S}(1 \mathrm{~s}) \rightarrow \sigma_{\mathrm{S}-\mathrm{C}}^{*}$} & \multirow{2}{*}{2474.4} & 2473.68 & 0.0075 & 2473.71 & 0.0075 \\
\hline & & & 2473.77 & 0.0070 & 2473.82 & 0.0068 \\
\hline
\end{tabular}

${ }^{a}$ Experimental excitation energies from Ref. 72.

${ }^{b}$ The splitting in the theoretical $\mathrm{S}(1 \mathrm{~s}) \rightarrow \sigma_{\mathrm{S}-\mathrm{S}}^{*}$ and $\mathrm{S}(1 \mathrm{~s}) \rightarrow \sigma_{\mathrm{S}-\mathrm{C}}^{*}$ excitations of cystine arises from breaking of the molecular symmetry during the geometry optimizations.

\section{Conclusions}

We have introduced time-dependent embedded mean-field theory (TD-EMFT) for the description of electronic excited states in complex systems. TD-EMFT is derived based on the time-dependent linear response formulation within the framework of embedded mean-field theory (EMFT). TD-EMFT offers potential advantages over conventional linear-response TDDFT in terms of the computational efficiency. Compared to other embedding approaches, TD-EMFT allows for straightforward implementation and calculation of nuclear gradients and transition properties. Furthermore, inherited from its parent ground-state method, TD-EMFT avoids the need to specify the number of electrons or spin state for each subsystem, allowing for the description of charge transfer and particle-number fluctuations between subsystems. We have demonstrated benchmarks of TD-EMFT for both local and non-local excitations in several organic molecules, as well as applications to chlorophyll a, solvatochromic shifts of pNA in explicit water, and sulfur K-edge XAS spectra of cysteine and cystine.

Overall, TD-EMFT gives excellent agreement with conventional linear-response TDDFT in terms of the excitation energy, oscillator strength, and orbital transition 


\section{Acknowledgments}

This material is based upon work performed by the Joint Center for Artificial Photosynthesis, a DOE Energy Innovation Hub, supported through the Office of Science of the U.S. Department of Energy under Award Number DE-SC0004993. T.F.M. and F.D. additionally acknowledge support from the Air Force Office of Scientific Research under Award Number FA9550-17-1-0102, and T.F.M. acknowledges support from a Camille Dreyfus Teacher-Scholar Award. F.R.M. and T.T. acknowledge support from the Engineering and Physical Sciences Research Council (EP/M013111/1).

\section{ASSOCIATED CONTENT}

(5) Supporting Information

Geometries in xyz format and specification of subsystem partitioning for all test molecules presented in this paper. This material is available free of charge via the Internet at http://pubs.acs.org.

\section{References}

(1) Dreuw, A.; Head-Gordon, M. Single-Reference Ab Initio Methods for the Calculation of Excited States of Large Molecules. Chem. Rev. 2005, 105, 4009-4037. 
(2) Casida, M. E. Time-Dependent Density-Functional Theory for Molecules and Molecular Solids. J. Mol. Struct. 2009, 914, 3-18.

(3) Krishtal, A.; Sinha, D.; Genova, A.; Pavanello, M. Subsystem density-functional theory as an effective tool for modeling ground and excited states, their dynamics and many-body interactions. J. Phys.-Condens. Mat. 2015, 27, 183202.

(4) Maitra, N. T. Perspective: Fundamental aspects of time-dependent density functional theory. J. Chem. Phys. 2016, 144, 220901.

(5) van Gisbergen, S. J.; Fonseca Guerra, C.; Baerends, E. J. Towards Excitation Energies and (Hyper) Polarizability Calculations of Large Molecules. Application of Parallelization and Linear Scaling Techniques to Time-Dependent Density Functional Response Theory. J. Comput. Chem. 2000, 21, 1511-1523.

(6) Coriani, S.; Høst, S.; Jansík, B.; Thøgersen, L.; Olsen, J.; Jørgensen, P.; Reine, S.; Pawłowski, F.; Helgaker, T.; Sałek, P. Linear-Scaling Implementation of Molecular Response Theory in Self-Consistent Field Electronic-Structure Theory. J. Chem. Phys. 2007, 126, 154108.

(7) Kjærgaard, T.; Jørgensen, P.; Olsen, J.; Coriani, S.; Helgaker, T. Hartree-Fock and Kohn-Sham Time-Dependent Response Theory in A Second-Quantization AtomicOrbital Formalism Suitable for Linear Scaling. J. Chem. Phys. 2008, 129, 054106.

(8) Yam, C.; Yokojima, S.; Chen, G. Linear-Scaling Time-Dependent DensityFunctional Theory. Phys. Rev. B 2003, 68, 153105.

(9) Cui, G.; Fang, W.; Yang, W. Reformulating Time-Dependent Density Functional Theory with Non-Orthogonal Localized Molecular Orbitals. Phys. Chem. Chem. Phys. 2010, 12, 416-421.

(10) Wu, F.; Liu, W.; Zhang, Y.; Li, Z. Linear-Scaling Time-Dependent Density Functional Theory Based on the Idea of "From Fragments to Molecule". J. Chem. Theor. Comput. 2011, 7, 3643-3660. 
(11) Liu, J.; Herbert, J. M. An Efficient and Accurate Approximation to TimeDependent Density Functional Theory for Systems of Weakly Coupled Monomers. J. Chem. Phys. 2015, 143, 034106.

(12) Liu, J.; Herbert, J. M. Local Excitation Approximations to Time-Dependent Density Functional Theory for Excitation Energies in Solution. J. Chem. Theor. Comput. 2015, 12, 157-166.

(13) Korona, T.; Werner, H.-J. Local Treatment of Electron Excitations in the EomCcsd Method. J. Chem. Phys. 2003, 118, 3006-3019.

(14) Chiba, M.; Fedorov, D. G.; Kitaura, K. Time-Dependent Density Functional Theory Based Upon the Fragment Molecular Orbital Method. J. Chem. Phys. 2007, 127, 104108.

(15) Casida, M. E.; Wesołowski, T. A. Generalization of the Kohn-Sham Equations with Constrained Electron Density Formalism and Its Time-Dependent Response Theory Formulation. Int. J. Quant. Chem. 2004, 96, 577-588.

(16) Neugebauer, J. Couplings Between Electronic Transitions in A Subsystem Formulation of Time-Dependent Density Functional Theory. J. Chem. Phys. 2007, 126, 134116.

(17) Gomes, a. S. P.; Jacob, C. R.; Visscher, L. Calculation of Local Excitations in Large Systems By Embedding Wave-Function Theory in Density-Functional Theory. Phys. Chem. Chem. Phys. 2008, 10, 5353-5362.

(18) Höfener, S.; Severo Pereira Gomes, a.; Visscher, L. Molecular Properties Via A Subsystem Density Functional Theory Formulation: A Common Framework for Electronic Embedding. J. Chem. Phys. 2012, 136, 044104.

(19) Neugebauer, J.; Louwerse, M. J.; Baerends, E. J.; Wesolowski, T. A. The Merits of the Frozen-Density Embedding Scheme to Model Solvatochromic Shifts. J. Chem. Phys. 2005, 122, 094115. 
(20) Chulhai, D. V.; Jensen, L. External Orthogonality in Subsystem Time-Dependent Density Functional Theory. Phys. Chem. Chem. Phys. 2016, 18, 21032-21039.

(21) Warshel, A.; Levitt, M. Theoretical Studies of Enzymic Reactions: Dielectric, Electrostatic and Steric Stabilization of the Carbonium Ion in the Reaction of Lysozyme. J. Mol. Biol. 1976, 103, 227-249.

(22) Gao, J. In Reviews in Computational Chemistry; Lipkowitz, K. B., Boyd, D. B., Eds.; VCH: New York, 1995; Vol. 7; pp 119-185.

(23) Sherwood, P. In Modern Methods and Algorithms of Quantum Chemistry; Grotendorst, J., Ed.; NIC Series; John von Neumann institute for Computing: Jülich, 2000; Vol. 1; pp 257-277.

(24) Senn, H. M.; Thiel, W. QM/MM Methods for Biomolecular Systems. Angew. Chem. Int. Ed. 2009, 48, 1198-1229.

(25) Goodpaster, J. D.; Ananth, N.; Manby, F. R.; Miller III, T. F. Exact Nonadditive Kinetic Potentials for Embedded Density Functional Theory. J. Chem. Phys. 2010, 133, 084103.

(26) Goodpaster, J. D.; Barnes, T. A.; Miller III, T. F. Embedded Density Functional Theory for Covalently Bonded and Strongly Interacting Subsystems. J. Chem. Phys. 2011, 134, 164108.

(27) Goodpaster, J. D.; Barnes, T. A.; Manby, F. R.; Miller III, T. F. Density Functional Theory Embedding for Correlated Wavefunctions: Improved Methods for Open-Shell Systems and Transition Metal Complexes. J. Chem. Phys. 2012, 13\%, 224113.

(28) Manby, F. R.; Stella, M.; Goodpaster, J. D.; Miller III, T. F. A Simple, Exact Density-Functional-Theory Embedding Scheme. J. Chem. Theor. Comput. 2012, $8,2564-2568$. 
(29) Barnes, T. A.; Goodpaster, J. D.; Manby, F. R.; Miller III, T. F. Accurate Basis Set Truncation for Wavefunction Embedding. J. Chem. Phys. 2013, 139, 024103.

(30) Goodpaster, J. D.; Barnes, T. A.; Manby, F. R.; Miller III, T. F. Accurate and Systematically Improvable Density Functional Theory Embedding for Correlated Wavefunctions. J. Chem. Phys. 2014, 140, 18 A507.

(31) Bennie, S. J.; Stella, M.; Miller III, T. F.; Manby, F. R. Accelerating Wavefunction in Density-Functional-Theory Embedding By Truncating the Active Basis Set. $J$. Chem. Phys. 2015, 143, 024105.

(32) Elliott, P.; Cohen, M. H.; Wasserman, A.; Burke, K. Density Functional Partition Theory with Fractional Occupations. J. Chem. Theor. Comput. 2009, 5, 827-833.

(33) Elliott, P.; Burke, K.; Cohen, M. H.; Wasserman, A. Partition Density-Functional Theory. Phys. Rev. A 2010, 82, 024501.

(34) Huang, C.; Carter, E. A. Potential-Functional Embedding Theory for Molecules and Materials. J. Chem. Phys. 2011, 135, 194104.

(35) Knizia, G.; Chan, G. K.-L. Density Matrix Embedding: A Simple Alternative to Dynamical Mean-Field Theory. Phys. Rev. Lett. 2012, 109, 186404.

(36) Knizia, G.; Chan, G. K.-L. Density Matrix Embedding: A Strong-Coupling Quantum Embedding Theory. J. Chem. Theor. Comput. 2013, 9, 1428-1432.

(37) Fornace, M. E.; Lee, J.; Miyamoto, K.; Manby, F. R.; Miller III, T. F. Embedded Mean-Field Theory. J. Chem. Theor. Comput. 2015, 11, 568-580.

(38) Ding, F.; Manby, F. R.; Miller III, T. F. Embedded Mean-Field Theory with Block-Orthogonalized Partitioning. J. Chem. Theor. Comput. 2017, 13, 16051615 . 
(39) Miyamoto, K.; Miller, T. F.; Manby, F. R. Fock-Matrix Corrections in Density Functional Theory and Use in Embedded Mean-Field Theory. J. Chem. Theor. Comput. 2016, 12, 5811-5822.

(40) Koh, K. J.; Nguyen-Beck, T. S.; Parkhill, J. Accelerating Realtime TDDFT with Block-Orthogonalized Manby-Miller Embedding Theory. J. Chem. Theor. Comput. 2017, in press, http://dx.doi.org/10.1021/acs.jctc.7b00494.

(41) Lee, S. J.; Miyamoto, K.; Ding, F.; Manby, F. R.; Miller III, T. F. Densitybased errors in mixed-basis mean-field electronic structure, with implications for embedding and QM/MM methods. Chem. Phys. Lett. 2017, 683, 375-382.

(42) Hehre, W. J.; Stewart, R. F.; Pople, J. A. Self-Consistent Molecular-Orbital Methods. I. Use of Gaussian Expansions of Slater-Type Atomic Orbitals. J. Chem. Phys. 1969, 51, 2657-64.

(43) Casida, M. E. In Recent Advances in Density Functional Methods, Part I; Chong, D., Ed.; World Scientific: Singapore, 1995; p 155.

(44) Manby, F. R.; Miller III, T. F. entos, Version 2016.1, An Ab Initio Molecular Simulation Package. 2016.

(45) Perdew, J. P.; Burke, K.; Ernzerhof, M. Generalized Gradient Approximation Made Simple. Phys. Rev. Lett. 1996, 77, 3865-3868.

(46) Becke, A. D. Density-Functional Exchange-Energy Approximation with Correct Asymptotic Behavior. Phys. Rev. A 1988, 38, 3098-3100.

(47) Stephens, P.; Devlin, F.; Chabalowski, C.; Frisch, M. Ab Initio Calculation of Vibrational Absorption and Circular Dichroism Spectra Using Density Functional Force Fields. J. Phys. Chem. 1994, 98, 11623.

(48) Polly, R.; Werner, H.-J.; Manby, F. R.; Knowles, P. J. Fast Hartree-Fock Theory Using Local Density Fitting Approximations. Mol. Phys. 2004, 102, 2311-2321. 
(49) Eichkorn, K.; Treutler, O.; Öhm, H.; Häser, M.; Ahlrichs, R. Auxiliary Basis Sets to Approximate Coulomb Potentials. Chem. Phys. Lett. 1995, 240, 283-290.

(50) Eichkorn, K.; Weigend, F.; Treutler, O.; Ahlrichs, R. Auxiliary Basis Sets for Main Row Atoms and Transition Metals and Their Use to Approximate Coulomb Potentials. Theor. Chem. Acc. 1997, 97, 119-124.

(51) Weigend, F. A Fully Direct Ri-Hf Algorithm: Implementation, Optimised Auxiliary Basis Sets, Demonstration of Accuracy and Efficiency. Phys. Chem. Chem. Phys. 2002, 4, 4285-4291.

(52) Frisch, M. J.; Trucks, G. W.; Schlegel, H. B.; Scuseria, G. E.; Robb, M. A.; Cheeseman, J. R.; Scalmani, G.; Barone, V.; Mennucci, B.; Petersson, G. A.; Nakatsuji, H.; Caricato, M.; Li, X.; Hratchian, H. P.; Izmaylov, A. F.; Bloino, J.; Zheng, G.; Sonnenberg, J. L.; Hada, M.; Ehara, M.; Toyota, K.; Fukuda, R.; Hasegawa, J.; Ishida, M.; Nakajima, T.; Honda, Y.; Kitao, O.; Nakai, H.; Vreven, T.; Montgomery, J. A., Jr.; Peralta, J. E.; Ogliaro, F.; Bearpark, M.; Heyd, J. J.; Brothers, E.; Kudin, K. N.; Staroverov, V. N.; Kobayashi, R.; Normand, J.; Raghavachari, K.; Rendell, A.; Burant, J. C.; Iyengar, S. S.; Tomasi, J.; Cossi, M.; Rega, N.; Millam, J. M.; Klene, M.; Knox, J. E.; Cross, J. B.; Bakken, V.; Adamo, C.; Jaramillo, J.; Gomperts, R.; Stratmann, R. E.; Yazyev, O.; Austin, A. J.; Cammi, R.; Pomelli, C.; Ochterski, J. W.; Martin, R. L.; Morokuma, K.; Zakrzewski, V. G.; Voth, G. A.; Salvador, P.; Dannenberg, J. J.; Dapprich, S.; Daniels, A. D.; Farkas, O.; Foresman, J. B.; Ortiz, J. V.; Cioslowski, J.; Fox, D. J. Gaussian 09 Revision A.1. Gaussian inc. Wallingford CT 2009.

(53) Vokacova, Z.; Burda, J. V. Computational Study on Spectral Properties of the Selected Pigments From Various Photosystems: Structure- Transition Energy Relationship. J. Phys. Chem. A 2007, 111, 5864-5878.

(54) Hasegawa, J.; Ozeki, Y.; Ohkawa, K.; Hada, M.; Nakatsuji, H. Theoretical Study 
of the Excited States of Chlorin, Bacteriochlorin, Pheophytin A, and Chlorophyll A By the Sac/Sac-Ci Method. J. Phys. Chem. B 1998, 102, 1320-1326.

(55) Parusel, a. B.; Grimme, S. A Theoretical Study of the Excited States of Chlorophyll A and Pheophytin A. J. Phys. Chem. B 2000, 104, 5395-5398.

(56) Linnanto, J.; Korppi-Tommola, J. Spectroscopic Properties of Mg-Chlorin, MgPorphin and Chlorophylls A, B, C 1, C 2, C 3 and D Studied By Semi-Empirical and Ab Initio Mo/Ci Methods. Phys. Chem. Chem. Phys. 2000, 2, 4962-4970.

(57) Linnanto, J.; Korppi-Tommola, J. Quantum Chemical Simulation of Excited States of Chlorophylls, Bacteriochlorophylls and Their Complexes. Phys. Chem. Chem. Phys. 2006, 8, 663-687.

(58) Cory, M. G.; Zerner, M. C.; Hu, X.; Schulten, K. Electronic Excitations in Aggregates of Bacteriochlorophylls. J. Phys. Chem. B 1998, 102, 7640-7650.

(59) Sundholm, D. Density Functional Theory Calculations of the Visible Spectrum of Chlorophyll A. Chem. Phys. Lett. 1999, 302, 480-484.

(60) König, C.; Neugebauer, J. First-Principles Calculation of Electronic Spectra of Light-Harvesting Complex II. Phys. Chem. Chem. Phys. 2011, 13, 10475-10490.

(61) König, C.; Neugebauer, J. Quantum Chemical Description of Absorption Properties and Excited-State Processes in Photosynthetic Systems. ChemPhysChem 2012, 13, 386-425.

(62) Neugebauer, J. Subsystem-Based Theoretical Spectroscopy of Biomolecules and Biomolecular Assemblies. ChemPhysChem 2009, 10, 3148-3173.

(63) Neugebauer, J. Chromophore-Specific Theoretical Spectroscopy: From Subsystem Density Functional Theory to Mode-Specific Vibrational Spectroscopy. Phys. Rep. 2010, 489, 1-87. 
(64) Isborn, C. M.; Mar, B. D.; Curchod, B. F.; Tavernelli, I.; Martinez, T. J. The Charge Transfer Problem in Density Functional Theory Calculations of Aqueously Solvated Molecules. J. Phys. Chem. B 2013, 117, 12189-12201.

(65) Lange, A.; Herbert, J. M. Simple Methods to Reduce Charge-Transfer Contamination in Time-Dependent Density-Functional Calculations of Clusters and Liquids. J. Chem. Theor. Comput. 2007, 3, 1680-1690.

(66) Li, J.; Ren, H.-S.; Ma, J.-Y.; Li, X.-Y. Spectral Shift of $\pi \rightarrow \pi^{*}$ Transition for P-Nitroaniline Based on A New Expression of Nonequilibrium Solvation Energy. Chinese J. Chem. Phys. 2014, 27, 181-188.

(67) Marenich, A. V.; Cramer, C. J.; Truhlar, D. G. Electronic Absorption Spectra and Solvatochromic Shifts By the Vertical Excitation Model: Solvated Clusters and Molecular Dynamics Sampling. J. Phys. Chem. B 2014, 119, 958-967.

(68) Kosenkov, D.; Slipchenko, L. V. Solvent Effects on the Electronic Transitions of P-Nitroaniline: A QM/EFP Study. J. Phys. Chem. A 2011, 115, 392-401.

(69) DeFusco, A.; Minezawa, N.; Slipchenko, L. V.; Zahariev, F.; Gordon, M. S. Modeling Solvent Effects on Electronic Excited States. J. Phys. Chem. Lett. 2011, 2, $2184-2192$.

(70) Sok, S.; Willow, S. Y.; Zahariev, F.; Gordon, M. S. Solvent-Induced Shift of the Lowest Singlet $\pi \rightarrow \pi^{*}$ Charge-Transfer Excited State of P-Nitroaniline in Water: An Application of the TDDFT/EFP1 Method. J. Phys. Chem. A 2011, 115, 9801-9809.

(71) Daday, C.; König, C.; Valsson, O.; Neugebauer, J.; Filippi, C. State-Specific Embedding Potentials for Excitation-Energy Calculations. J. Chem. Theor. Comput. 2013, 9, 2355-2367.

(72) Rompel, A.; Cinco, R. M.; Latimer, M. J.; McDermott, A. E.; Guiles, R.; Quintanilha, A.; Krauss, R. M.; Sauer, K.; Yachandra, V. K.; Klein, M. P. Sulfur 
K-Edge X-Ray Absorption Spectroscopy: A Spectroscopic Tool to Examine the Redox State of S-Containing Metabolites in vivo. Proc. Natl. Acad. Sci. U.S.A. 1998, 95, 6122-6127.

(73) Hua, W.; Gao, B.; Li, S.; Ågren, H.; Luo, Y. Refinement of DNA Structures Through Near-Edge X-Ray Absorption Fine Structure Analysis: Applications on Guanine and Cytosine Nucleobases, Nucleosides, and Nucleotides. J. Phys. Chem. B 2010, 114, 13214-13222.

(74) Yano, J.; Yachandra, V. K. X-Ray Absorption Spectroscopy. Photosynth. Res. 2009, 102, 241.

(75) Penner-Hahn, J. E. Characterization of "Spectroscopically Quiet" Metals in Biology. Coordin. Chem. Rev. 2005, 249, 161-177.

(76) Nilsson, a.; Pettersson, L. G. M. Chemical Bonding on Surfaces Probed By X-Ray Emission Spectroscopy and Density Functional Theory. Surf. Sci. Rep. 2004, 55, 49-167.

(77) Hähner, G. Near Edge X-Ray Absorption Fine Structure Spectroscopy As A Tool to Probe Electronic and Structural Properties of Thin Organic Films and Liquids. Chem. Soc. Rev. 2006, 35, 1244-1255.

(78) Contini, G.; Carravetta, V.; Di Castro, V.; Stranges, S.; Richter, R.; Alagia, M. Gas-Phase Experimental and Theoretical Near Edge X-Ray Absorption Fine Structure Study of 2-Mercaptobenzothiazole. J. Phys. Chem. A 2001, 105, 73087314 .

(79) Liang, W.; Fischer, S. A.; Frisch, M. J.; Li, X. Energy-Specific Linear Response TDHF/TDDFT for Calculating High-Energy Excited States. J. Chem. Theor. Comput. 2011, 7, 3540-3547. 
(80) Lopata, K.; Van Kuiken, B. E.; Khalil, M.; Govind, N. Linear-Response and RealTime Time-Dependent Density Functional Theory Studies of Core-Level NearEdge X-Ray Absorption. J. Chem. Theor. Comput. 2012, 8, 3284-3292.

(81) Stener, M.; Fronzoni, G.; De Simone, M. Time Dependent Density Functional Theory of Core Electrons Excitations. Chem. Phys. Lett. 2003, 373, 115-123.

(82) Besley, N. A.; Asmuruf, F. A. Time-Dependent Density Functional Theory Calculations of the Spectroscopy of Core Electrons. Phys. Chem. Chem. Phys. 2010, 12, 12024-12039.

(83) Besley, N. A.; Gilbert, a. T.; Gill, P. M. Self-Consistent-Field Calculations of Core Excited States. J. Chem. Phys. 2009, 130, 124308.

(84) Derricotte, W. D.; Evangelista, F. A. Simulation of X-Ray Absorption Spectra with Orthogonality Constrained Density Functional Theory. Phys. Chem. Chem. Phys. 2015, 17, 14360-14374.

(85) Prendergast, D.; Galli, G. X-Ray Absorption Spectra of Water From First Principles Calculations. Phys. Rev. Lett. 2006, 96, 215502.

(86) Sheehy, J.; Gil, T.; Winstead, C.; Farren, R.; Langhoff, P. Correlation of Molecular Valence-And K-Shell Photoionization Resonances with Bond Lengths. J. Chem. Phys. 1989, 91, 1796-1812.

(87) Coriani, S.; Christiansen, O.; Fransson, T.; Norman, P. Coupled-Cluster Response Theory for Near-Edge X-Ray-Absorption Fine Structure of Atoms and Molecules. Phys. Rev. A 2012, 85, 022507.

(88) Schirmer, J. Beyond the Random-Phase Approximation: A New Approximation Scheme for the Polarization Propagator. Phys. Rev. A 1982, 26, 2395.

(89) Trofimov, A.; Schirmer, J. An Efficient Polarization Propagator Approach to Valence Electron Excitation Spectra. J. Phys. B: At. Mol. Opt. Phys. 1995, 28, 2299. 
(90) Risberg, E. D.; Jalilehvand, F.; Leung, B. O.; Pettersson, L. G.; Sandström, M. Theoretical and Experimental Sulfur K-Edge X-Ray Absorption Spectroscopic Study of Cysteine, Cystine, Homocysteine, Penicillamine, Methionine and Methionine Sulfoxide. Dalton Trans. 2009, 3542-3558.

(91) Besley, N. A.; Peach, M. J.; Tozer, D. J. Time-Dependent Density Functional Theory Calculations of Near-Edge X-Ray Absorption Fine Structure with ShortRange Corrected Functionals. Phys. Chem. Chem. Phys. 2009, 11, 10350-10358. 


\section{TOC Graphic}
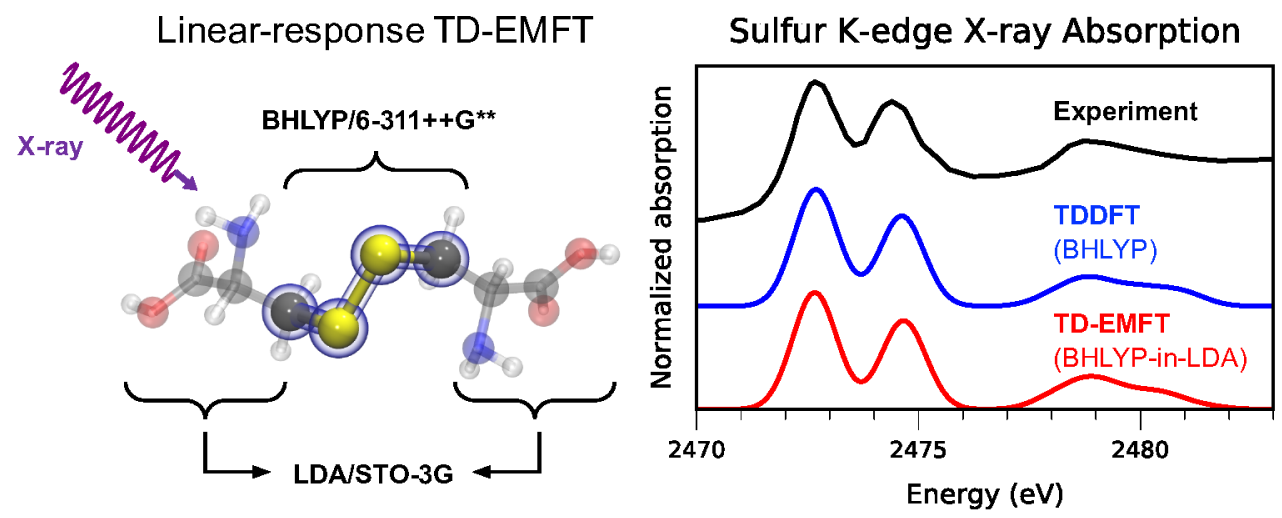

19

20

21

22

23

24

25

26

27

28

29

30

31

32

33

34

35

36

37

38

39

40

41

42

43

44

45

46

47

48

49

50

51

52

53

54

55

56

57

58

59

60 